\title{
Redefining the T1 category of the 8th American Joint Committee on Cancer Staging System for intrahepatic cholangiocarcinoma: A SEER database analysis
}

\author{
YANG LI $^{1}$, CHUANGANG TANG ${ }^{2}$, BINGQING ZHAO $^{3}$ and FANG DONG ${ }^{4}$ \\ ${ }^{1}$ Hepatobiliary Surgery Department, Organ Transplantation Center, Tianjin First Central Hospital, Tianjin 300192; \\ ${ }^{2}$ Department of Breast Surgery, Xuzhou Central Hospital, The Affiliated Xuzhou Hospital of Medical College of \\ Southeast University, Xuzhou, Jiangsu 221009; ${ }^{3}$ Department of Surgery, Tianjin Second People's Hospital, Tianjin 300192; \\ ${ }^{4}$ Vascular Surgery Department, Gansu Provincial Hospital, Lanzhou, Gansu 730000, P.R. China
}

Received March 13, 2019; Accepted September 9, 2019

DOI: $10.3892 / \mathrm{ol} .2019 .11096$

\begin{abstract}
The T1 tumor category is further divided into T1a $(\leq 5 \mathrm{~cm})$ and $\mathrm{Tlb}(>5 \mathrm{~cm})$ by tumor size in the 8 th edition of the American Joint Committee on Cancer (AJCC) staging system for intrahepatic cholangiocarcinoma (ICC). The aim of the present study was to investigate the association between tumor size and prognosis in patients with T1 ICC. The data regarding patients with ICC was downloaded from the Surveillance, Epidemiology, and End Results database between January 2004 and December 2013, at the time of first diagnosis and entered into the database. Demographic and pathological characteristics of patients were analyzed using an independent $t$-test and $\chi^{2}$ test. Overall survival was evaluated using Kaplan-Meier analysis; the cut-off point for tumor size was determined using the X-tile software. A total of 407 patients with ICC were selected from the analysis, including 199 cases with stage IA and 208 cases with stage IB tumors. The independent prognostic factors for patients with stage IA ICC were age and surgery. Independent prognostic factors for patients with stage IB ICC included age, surgery (yes vs. no) and tumor size $(5-7 \mathrm{vs} . \geq 7 \mathrm{~cm})$. The optimal cut-off value for tumor size was determined to be $\sim 7 \mathrm{~cm}$ using X-tile software. Tumor size with a cut-off value of $7 \mathrm{~cm}$ could stratify patients by risk better than a value of $5 \mathrm{~cm}$ [hazard ratio (HR), 1.775; 95\% confidence interval (CI), 1.356-2.323 and; HR, $1.402 ; 95 \%$ CI, 1.078-1.824, respectively]. This suggests that the T1 tumor category should be subclassified into T1a and T1b with a cut-off of $7 \mathrm{~cm}$ rather than $5 \mathrm{~cm}$. The next edition of the AJCC staging system may take the present evidence into consideration for improvement regarding the accurate staging of ICC.
\end{abstract}

Correspondence to: Dr Fang Dong, Vascular Surgery Department, Gansu Provincial Hospital, 204 Donggang West Road, Lanzhou, Gansu 730000, P.R. China

E-mail: 187248578@qq.com

Key words: intrahepatic cholangiocarcinoma, staging, tumor size, overall survival

\section{Introduction}

Intrahepatic bile duct cancer (IHBDC) is a type of cancer usually considered to be a primary liver malignancy (1). The most common histological type of IHBDC is intrahepatic cholangiocarcinoma (ICC), with a 5-year overall survival (OS) rate of $15-45 \%$ worldwide (2-5). Considering these poor patient outcomes, an accurate staging system is required to stratify patients by risk of mortality.

Recently, the 8th edition of American Joint Committee on Cancer (AJCC) staging system for ICC has been released and applied in clinical practice (6). The main changes focus on the definition of T category. Of these, the T1 category was further divided into $\mathrm{T} 1 \mathrm{a}(\leq 5 \mathrm{~cm})$ and $\mathrm{T} 1 \mathrm{~b}(>5 \mathrm{~cm})$ by tumor size. This is the first time the impact of tumor size in the AJCC staging system has been accounted for.

Yamashita et al (7) identified that patients with ICC and a tumor size $\geq 4.4 \mathrm{~cm}$ were more likely to relapse compared with patients with ICC and a tumor size $<4.4 \mathrm{~cm}$. Furthermore, Spolverato et al (8) reported that the larger the tumor size, the higher the incidence of microscopic vascular invasion. In addition, larger tumors were associated with poor biological behaviors (e.g. worse tumor grade) (8). These findings suggest that large tumor sizes have a negative impact on the survival of patients with ICC. However, the rationality of the cut-off value of $5 \mathrm{~cm}$ has not yet been validated in the latest AJCC staging system.

In the present study, the Surveillance Epidemiology and End Results (SEER) database, a population-based database, was used to assess the relationship between the tumor size and prognosis. Specifically, the optimal cut-off value for tumor size in the stratification of T1 ICC tumors was investigated.

\section{Materials and methods}

Patients. Patient data were downloaded from the SEER database (https:seer.cancer.gov) (between Jan 2004 and Dec 2013, during first diagnosis and entered into the database) using the SEER*Stat software (version 8.2.0; National Cancer Institute). ICC was retrieved from the site recode C22.1, according to 
Table I. Demographic and pathological characteristics of patients with intrahepatic cholangiocarcinoma.

\begin{tabular}{|c|c|c|c|c|}
\hline Variables & Overall $(n=407)$ & Stage IA $(n=199)$ & Stage IB $(n=208)$ & P-value \\
\hline Age, years ${ }^{\mathrm{a}}$ & $66(18-99)$ & $66(35-97)$ & 67 (18-99) & $>0.05$ \\
\hline Ethnicity & & & & $>0.05$ \\
\hline Caucasian & 297 & 146 & 151 & \\
\hline African American & 40 & 16 & 24 & \\
\hline Other & 70 & 37 & 33 & \\
\hline Sex & & & & $>0.05$ \\
\hline Male & 183 & 95 & 88 & \\
\hline Female & 224 & 104 & 120 & \\
\hline Marital status & & & & 0.027 \\
\hline Married & 231 & 124 & 107 & \\
\hline Other $^{b}$ & 176 & 75 & 101 & \\
\hline Tumor size, $\mathrm{mm}^{\mathrm{c}}$ & $58.1(5.0-167.0)$ & $33.2(5.0-50.0)$ & $82.0(51.0-167.0)$ & $<0.001$ \\
\hline Grade & & & & $>0.05$ \\
\hline $\mathrm{I}+\mathrm{II}$ & 193 & 102 & 91 & \\
\hline III+IV & 85 & 37 & 48 & \\
\hline Unknown & 129 & 60 & 69 & \\
\hline Surgery & & & & $<0.001$ \\
\hline Yes & 230 & 128 & 102 & \\
\hline No & 177 & 71 & 106 & \\
\hline
\end{tabular}

aPresented as median (range). bincludes those that are divorced, separated, single (never married), widowed and unknown marital status. 'Presented as mean (range).

the International Classification of Diseases for Oncology (3rd edition) (9). The inclusion criteria were as follows: i) Age, $\geq 18$ years; ii) diagnosed as ICC with positive histology confirmed by histopathological and immunohistochemical analysis; iii) stage I tumor according to the 8th AJCC TNM staging system (10); iv) definite tumor size stated in mm; v) first primary tumor; and vi) available follow-up information. Demographic and pathological characteristics included age, ethnicity, sex, marital status [classified as married or other, (divorced, separated, single (never married), widowed and unknown marital status)], tumor size, grade and surgical treatment. The total follow-up time ranged between 0 and 118 months.

Statistical analysis. The differences between patients with stage IA and IB tumors were evaluated using an independent $\mathrm{t}$-test or $\chi^{2}$ test. Univariate and multivariate analyses were performed using the Cox regression model and the data is presented as hazard ratio (HR) and $95 \%$ confidence interval (CI). Patients with unknown grade information were excluded in the univariate and multivariate analyses. Survival curves were then plotted using the Kaplan-Meier method and the log-rank test. X-tile software (version 3.6.1; Yale University) was used to examine the optimal tumor size (11). All analyses were performed using PASW statistics v18 (SPSS, Inc). P<0.05 was considered to indicate a statistically significant difference.

\section{Results}

A total of 407 patients with ICC, including 199 cases with stage IA and 208 cases with stage IB tumors, were finally selected

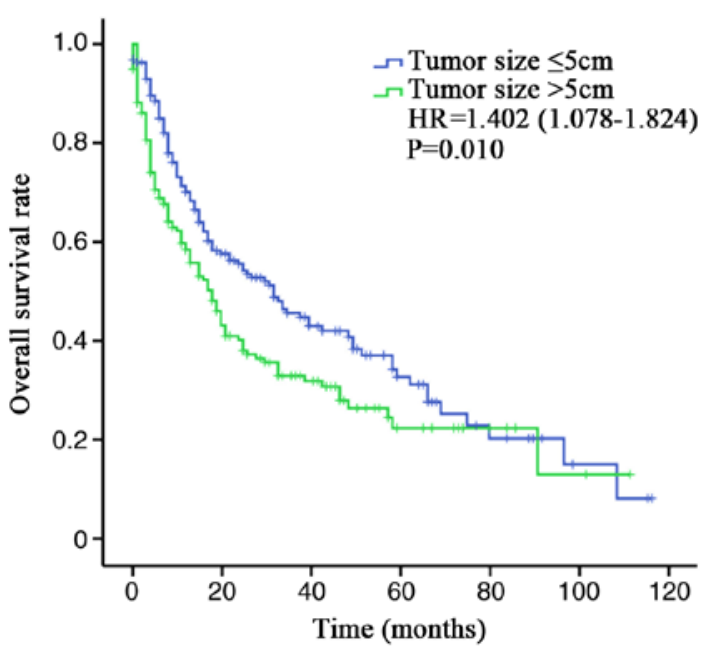

Figure 1. Survival curves of patients with stage I intrahepatic cholangiocarcinoma using the tumor size sub-classification cut-off value of $5 \mathrm{~cm}$.

for further analysis using inclusion criteria. Demographic and pathological characteristics are presented in Table I. The median age of the entire cohort was 66 years (range, 18-99 years), and the majority of patients were Caucasian (73.0\%). The mean tumor size was $58.1 \mathrm{~mm}$ (range, 5-167). Additionally, the proportion of patients with well- and moderately differentiated tumors (grade I+II) was higher compared with those with poorly differentiated and undifferentiated tumors (grade III+IV) (47.4 vs. 20.9\%). Except for patients with unknown grade information, the incidence of grade III+IV 
Table II. Univariate Cox regression model analysis of overall survival in patients with intrahepatic cholangiocarcinoma.

\begin{tabular}{|c|c|c|c|c|}
\hline \multirow[b]{2}{*}{ Variables } & \multicolumn{2}{|c|}{ Stage IA } & \multicolumn{2}{|c|}{ Stage IB } \\
\hline & HR $(95 \% \mathrm{CI})$ & P-value & $\mathrm{HR}(95 \% \mathrm{CI})$ & P-value \\
\hline Age, years & $1.036(1.016-1.055)$ & $<0.001$ & $1.039(1.023-1.056)$ & $<0.001$ \\
\hline \multicolumn{5}{|l|}{ Ethnicity } \\
\hline Caucasian & Reference & & Reference & \\
\hline African American & $1.032(0.516-2.062)$ & 0.929 & $1.044(0.593-1.838)$ & 0.882 \\
\hline Other & $0.744(0.457-1.211)$ & 0.234 & $1.131(0.671-1.905)$ & 0.644 \\
\hline \multicolumn{5}{|l|}{ Sex } \\
\hline Male & Reference & & Reference & \\
\hline Female & $1.023(0.699-1.499)$ & 0.905 & $0.764(0.530-1.101)$ & 0.148 \\
\hline \multicolumn{5}{|l|}{ Grade } \\
\hline $\mathrm{I}+\mathrm{II}$ & Reference & & Reference & \\
\hline III+IV & $1.680(1.031-2.735)$ & 0.037 & $1.849(1.158-2.952)$ & 0.010 \\
\hline \multicolumn{5}{|l|}{ Marital status } \\
\hline Married & Reference & & Reference & \\
\hline Other & $1.086(0.731-1.613)$ & 0.682 & $1.092(0.761-1.567)$ & 0.634 \\
\hline \multicolumn{5}{|l|}{ Surgery } \\
\hline Yes & Reference & & Reference & \\
\hline No & $4.178(2.820-6.188)$ & $<0.001$ & $4.071(2.732-6.066)$ & $<0.001$ \\
\hline \multicolumn{5}{|l|}{ Tumor size, mm } \\
\hline $51-69$ & & & Reference & \\
\hline$\geq 70$ & - & - & $1.882(1.242-2.851)$ & 0.003 \\
\hline
\end{tabular}

HR, hazard ratio; CI, confidence interval.

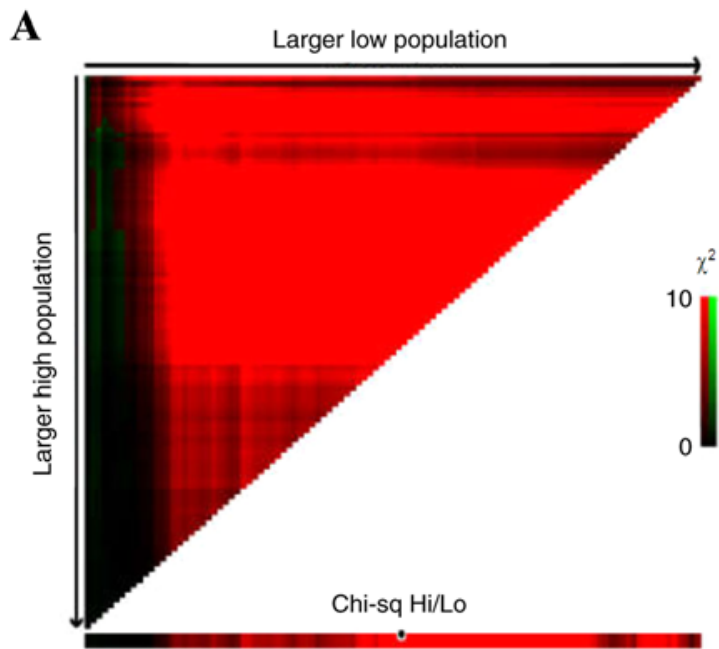

B

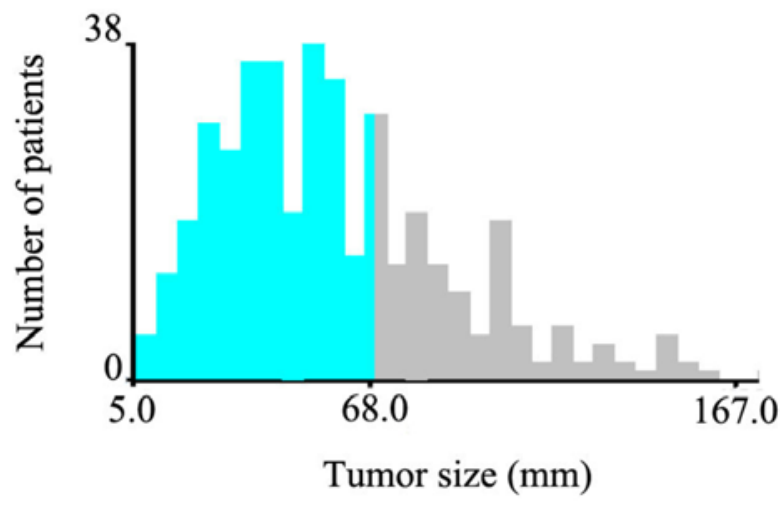

Figure 2. Optimal cut-off point determined using X-tile software. (A) X-tile plots are created by dividing tumor size into two populations randomly: low and high. All possible cut-off points were assessed. The brightest pixel (black/white circle on the $\chi^{2} \mathrm{Hi} / \mathrm{Lo}$ axis) denotes the optimal cut-off point. $\chi^{2}$ values are indicated by the red and black colours. (B) Histogram of the entire cohort. The blue area represents patients with tumor size $\leq 68 \mathrm{~mm}$; the grey area represents patients with tumor size $>68 \mathrm{~mm}$. Hi, high; Li, low.

was significantly higher in stage IB than in stage IA (34.5 vs. 26.6\%). There were no significant differences between stage IA and IB with respect to patient age, ethnicity, sex and grade $(\mathrm{P}>0.05)$. However, significantly more patients with stage IA received surgical treatment compared with patients with stage IB (64.3 vs. $49.0 \%$; $\mathrm{P}<0.001)$. Patients who were married also accounted for a higher proportion of patients with stage IA compared with stage IB $(\mathrm{P}<0.027)$. 
Table III. Multivariate Cox regression model analysis of overall survival in patients with intrahepatic cholangiocarcinoma.

\begin{tabular}{|c|c|c|c|c|}
\hline \multirow[b]{2}{*}{ Variables } & \multicolumn{2}{|c|}{ Stage IA } & \multicolumn{2}{|c|}{ Stage IB } \\
\hline & $\mathrm{HR}(95 \% \mathrm{CI})$ & P-value & $\mathrm{HR}(95 \% \mathrm{CI})$ & P-value \\
\hline Age, years & $1.027(1.008-1.046)$ & 0.005 & $1.034(1.017-1.052)$ & $<0.001$ \\
\hline \multicolumn{5}{|l|}{ Ethnicity } \\
\hline Caucasian & Reference & & Reference & \\
\hline African American & $1.165(0.580-2.340)$ & 0.668 & $1.045(0.583-1.870)$ & 0.883 \\
\hline Other & $0.699(0.426-1.145)$ & 0.155 & $0.946(0.553-1.617)$ & 0.839 \\
\hline \multicolumn{5}{|l|}{ Sex } \\
\hline Male & Reference & & Reference & \\
\hline Female & $0.909(0.597-1.386)$ & 0.658 & $0.877(0.593-1.295)$ & 0.509 \\
\hline \multicolumn{5}{|l|}{ Grade } \\
\hline $\mathrm{I}+\mathrm{II}$ & Reference & & Reference & \\
\hline $\mathrm{III}+\mathrm{IV}$ & $1.315(0.797-2.170)$ & 0.284 & $1.643(1.000-2.697)$ & 0.050 \\
\hline \multicolumn{5}{|l|}{ Surgery } \\
\hline Yes & Reference & & Reference & \\
\hline No & $3.713(2.431-5.670)$ & $<0.001$ & $2.765(1.795-4.259)$ & $<0.001$ \\
\hline \multicolumn{5}{|l|}{ Tumor size, mm } \\
\hline $51-69$ & & & Reference & \\
\hline$\geq 70$ & - & - & $1.757(1.134-2.724)$ & 0.012 \\
\hline
\end{tabular}

HR, hazard ratio; CI, confidence interval.

Univariate and multivariate Cox regression model analyses of OS time were performed for patients with ICC (Tables II and III). In univariate analysis, prognostic factors identified in patients with stage IA ICC included age, grade (I+II vs. III+IV) and surgery (yes vs. no). Prognostic factors identified in patients with stage IB ICC included age, grade (I+II vs. III+IV), surgery (yes vs. no) and tumor size (5-7 vs. $\geq 7 \mathrm{~cm}$ ). In multivariate analysis, independent prognostic factors identified in patients with stage IA ICC were age and surgery (yes vs. no). Alternatively, independent prognostic factors for patients with stage IB ICC included age, surgery (yes vs. no) and tumor size (5-7 vs. $\geq 7 \mathrm{~cm}$ ).

According to the current AJCC TNM staging system, patients with stage IA had a significantly longer survival time compared with that in patients with stage IB (Fig. 1; $\mathrm{P}=0.010$ ). $\mathrm{X}$-tile software was used to investigate the association between tumor size and risk of mortality (Fig. 2A and B). The plots are created by dividing tumor size into two populations, randomly: low and high. All possible cut-off points were assessed. The brightest pixel (indicated by the black/white circle on the $\chi^{2}$ high/low axis) denotes the optimal cut-off point. As a result, the optimal cut-off value of tumor size was shown to be $6.8 \mathrm{~cm}$. Thus $7 \mathrm{~cm}$ was used as an integer divider to redefine the subclassifications of $\mathrm{T} 1(\mathrm{~T} 1 \mathrm{a},<7 \mathrm{~cm}$; T1b, $\geq 7 \mathrm{~cm}$ ). The survival curves of modified stage IA and IB are presented in Fig. $3(\mathrm{P}<0.001)$. Tumor size with a cut-off value of $7 \mathrm{~cm}$ could better stratify patients by risk compared with $5 \mathrm{~cm}$ (7 cm, HR, 1.775; 95\% CI, 1.356-2.323; $5 \mathrm{~cm}$, HR, 1.402; 95\% CI, 1.078-1.824).

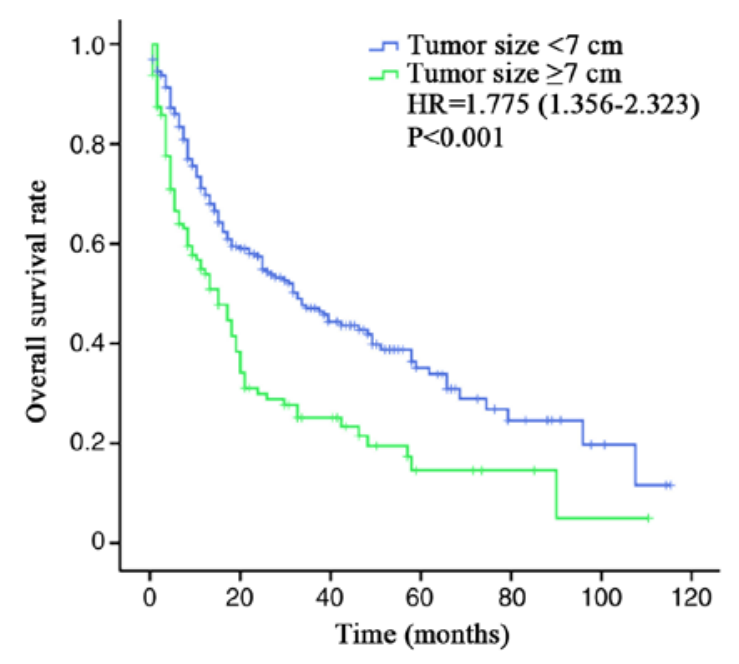

Figure 3. Survival curves of patients with stage I intrahepatic cholangiocarcinoma using the tumor size subclassification cut-off value of $7 \mathrm{~cm}$.

\section{Discussion}

ICC is a relatively uncommon cancer; however, some patients miss their interventional operative opportunity due to delayed diagnosis (12). The present study demonstrated that the proportion of patients with stage IA receiving operations was much higher than those with stage IB (64.3 vs. $49.0 \%$ ), indicating that tumor size was a prominent factor in decisions 
about surgical treatment. Besides surgery, the other common prognostic factor for both stage IA and IB was age, which was in agreement with many previous studies (13-16). Age was significantly associated with OS time (Table III).

Notably, poor tumor differentiation has also been considered to be a risk factor for ICC in several studies with large sample sizes (16-18). Spolverato et al (8) identified that the incidence of poor tumor differentiation was significantly higher in large tumors $(<3 \mathrm{~cm}, 9.7 \% ; 3-5 \mathrm{~cm}, 19.8 \% ; 5-7 \mathrm{~cm}, 24.2 \%$; $7-15 \mathrm{~cm}, 21.1 \%$; >15 cm, 31.6\%), which was similar to the present results (Table I). The present study indicated that grade was not an independent prognostic factor for patients with stage IA ( $\mathrm{P}=0.284$; Table III). However, grade was nearly an independent prognostic factor for patients with stage IB $(\mathrm{P}=0.05$; Table III). This is likely explained by the high proportion of patients with stage IA receiving surgery, resulting in better outcomes, regardless of the degree of tumor differentiation.

Survival outcomes were significantly different between stage IA and IB, suggesting the sub-classification of stage I by tumor size is necessary. The present findings demonstrated the most appropriate cut-off value for tumor size is $7 \mathrm{~cm}$ rather than $5 \mathrm{~cm}$. In a large, multi-institutional study, Hyder et al (19) also reported that the impact of tumor size on the risk of mortality plateaued at a threshold value of $7 \mathrm{~cm}$ in patients with resectable ICC. These findings suggested that the optimal size cut-off value of the T1 category in the 8th edition of AJCC staging system was worth considering to better predict the outcomes of patients with ICC.

It should be noted that the present study was limited by using retrospective clinical data. For this reason, the optimal cut off value of $7 \mathrm{~cm}$ should be validated by external data and therefore more prospective studies should be performed in a larger cohort of patients with ICC in future research. A total of 129 cases with unknown grade information were excluded in the univariate and multivariate Cox regression analysis, which may impact the results to some extent. Additionally, the SEER database does not include genetic information of tumors. Therefore, the potential mechanism of large tumor size leading to poor prognosis remains to be elucidated through further basic laboratory research.

\section{Acknowledgements}

Not applicable.

\section{Funding}

The present study was supported by the Tianjin Municipal Health Bureau of Science and Technology Fund Projects (grant no. 2015KZ034).

\section{Availability of data and materials}

The datasets used and/or analyzed during the present study are available from the SEER database (https://seer.cancer.gov/).

\section{Authors' contributions}

FD and YL designed the research and critically revised the manuscript for important intellectual content. CT and BZ carried out administrative support and performed the collection, assembly and interpretation of data. All authors performed the research, analyzed the data and wrote the paper.

\section{Ethics approval and consent to participate}

This study was approved by the institutional review board of Gansu Provincial Hospital, China.

\section{Patient consent for publication}

Not applicable.

\section{Competing interests}

The authors declare that they have no competing interests.

\section{References}

1. Ikai I, Arii S, Okazaki M, Okita K, Omata M, Kojiro M, Takayasu K, Nakanuma Y, Makuuchi M, Matsuyama Y, et al: Report of the 17th Nationwide Follow-up Survey of Primary Liver Cancer in Japan. Hepatol Res 37: 676-691, 2007.

2. Hu J, Chen FY, Zhou KQ, Zhou C, Cao Y, Sun HC, Fan J, Zhou J and Wang Z: Intrahepatic cholangiocarcinoma patients without indications of lymph node metastasis not benefit from lymph node dissection. Oncotarget 8: 113817-113827, 2017.

3. Aljiffry M, Abdulelah A, Walsh M, Peltekian K, Alwayn I and Molinari M: Evidence-based approach to cholangiocarcinoma: A systematic review of the current literature. J Am Coll Surg 208: 134-147, 2009.

4. Poultsides GA, Zhu AX, Choti MA and Pawlik TM: Intrahepatic cholangiocarcinoma. Surg Clin North Am 90: 817-837, 2010.

5. Lin XH and Luo JC: The risk factors and prognostic factors of intrahepatic cholangiocarcinoma. J Chin Med Assoc 80: 121-122, 2017.

6. Amin MB, Greene FL, Edge SB, Compton CC, Gershenwald JE, Brookland RK, Meyer L, Gress DM, Byrd DR and Winchester DP: The Eighth Edition AJCC Cancer Staging Manual: Continuing to build a bridge from a population-based to a more 'personalized' approach to cancer staging. CA Cancer J Clin 67: 93-99 2017.

7. Yamashita YI, Shirabe K, Beppu T, Eguchi S, Nanashima A, Ohta M, Ueno S, Kondo K, Kitahara K, Shiraishi M, et al: Surgical management of recurrent intrahepatic cholangiocarcinoma: predictors, adjuvant chemotherapy, and surgical therapy for recurrence: A multi-institutional study by the Kyushu Study Group of Liver Surgery. Ann Gastroenterol Surg 1: 136-142, 2017.

8. Spolverato G, Ejaz A, Kim Y, Sotiropoulos GC, Pau A, Alexandrescu S, Marques H,Pulitano C, Barroso E, Clary BM, et al: Tumor size predicts vascular invasion and histologic grade among patients undergoing resection of intrahepatic cholangiocarcinoma. J Gastrointest Surg 18: 1284-1291, 2014.

9. Fritz A, Percy C, Jack A, Shanmugaratnam K, Sobin L, Parkin DM (eds): International classification of diseases for oncology (ICD-O), 3ed. Geneva: World Health Organization, 2013.

10. Lee AJ and Chun YS: Intrahepatic cholangiocarcinoma: The AJCC/UICC 8th edition updates. Chin Clin Oncol 7: 52, 2018.

11. Camp RL, Dolled-Filhart $M$ and Rimm DL: X-tile: A new bio-informatics tool for biomarker assessment and outcome-based cut-point optimization. Clin Cancer Res 10: 7252-7259, 2004.

12. Kim Y, Moris DP, Zhang XF, Bagante F, Spolverato G, Schmidt C, Dilhoff $M$ and Pawlik TM: Evaluation of the 8th edition American Joint Commission on Cancer (AJCC) staging system for patients with intrahepatic cholangiocarcinoma: A surveillance, epidemiology, and end results (SEER) analysis. J Surg Oncol 116: 643-650, 2017.

13. Wang Y, Li J, Xia Y, Gong R, Wang K, Yan Z, Wan X, Liu G, Wu D, Shi L, et al: Prognostic nomogram for intrahepatic cholangiocarcinoma after partial hepatectomy. J Clin Oncol 31: 1188-1195, 2013.

14. Clark CJ, Wood-Wentz CM, Reid-Lombardo KM, Kendrick ML, Huebner $\mathrm{M}$ and Que FG: Lymphadenectomy in the staging and treatment of intrahepatic cholangiocarcinoma: A population-based study using the National Cancer Institute SEER database. HPB (Oxford) 13: 612-620, 2011. 
15. Bunsiripaiboon $P$, Sornmayura $P$, Wilasrusmee $C$ and Lertsithichai P: The prognostic significance of microvessel density in intrahepatic cholangiocarcinoma. J Med Assoc Thai 93: 66-72, 2010.

16. Tamandl D, Herberger B, Gruenberger B, Puhalla H, Klinger M and Gruenberger T: Influence of hepatic resection margin on recurrence and survival in intrahepatic cholangiocarcinoma. Ann Surg Oncol 15: 2787-2794, 2008.

17. Ribero D, Pinna AD, Guglielmi A, Ponti A, Nuzzo G, Giulini SM, Aldrighetti L, Calise F, Gerunda GE, Tomatis M, et al: Surgical approach for long-term survival of patients with intrahepatic cholangiocarcinoma: A multi-institutional analysis of 434 patients. Arch Surg 147: 1107-1113, 2012.
18. Fisher SB, Patel SH, Kooby DA, Weber S, Bloomston M, Cho C, Hatzaras I, Schmidt C, Winslow E, Staley CA III, et al. Lymphovascular and perineural invasion as selection criteria for adjuvant therapy in intrahepatic cholangiocarcinoma: A multi-institution analysis. HPB (Oxford) 14: 514-522, 2012.

19. Hyder O, Marques H, Pulitano C, Marsh JW, Alexandrescu S, Bauer TW, Gamblin TC, Sotiropoulos GC, Paul A, Barroso E, et al: A nomogram to predict long-term survival after resection for intrahepatic cholangiocarcinoma: An Eastern and Western experience. JAMA Surg 149: 432-438, 2014. 